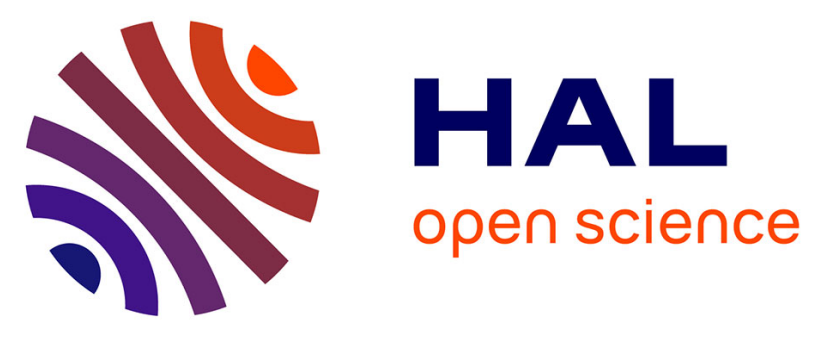

\title{
Black Hole Growth and Active Galactic Nuclei Obscuration by Instability-Driven Inflows in High-Redshift Disk Galaxies Fed by Cold Streams
}

Frédéric Bournaud, Avishai Dekel, Romain Teyssier, Marcello Cacciato, Emanuele Daddi, Stéphanie Juneau, Francesco Shankar

\section{To cite this version:}

Frédéric Bournaud, Avishai Dekel, Romain Teyssier, Marcello Cacciato, Emanuele Daddi, et al.. Black Hole Growth and Active Galactic Nuclei Obscuration by Instability-Driven Inflows in HighRedshift Disk Galaxies Fed by Cold Streams. The Astrophysical journal letters, 2011, 741, pp.L33. 10.1088/2041-8205/741/2/L33 . cea-00826998

\section{HAL Id: cea-00826998 https: / hal-cea.archives-ouvertes.fr/cea-00826998}

Submitted on 30 Nov 2020

HAL is a multi-disciplinary open access archive for the deposit and dissemination of scientific research documents, whether they are published or not. The documents may come from teaching and research institutions in France or abroad, or from public or private research centers.
L'archive ouverte pluridisciplinaire HAL, est destinée au dépôt et à la diffusion de documents scientifiques de niveau recherche, publiés ou non, émanant des établissements d'enseignement et de recherche français ou étrangers, des laboratoires publics ou privés. 


\title{
University of Zurich $^{\text {vit }}$
}

\section{Black hole growth and active galactic nuclei obscuration by instability-driven inflows in high-redshift disk galaxies fed by cold streams}

\author{
Bournaud, F ; Dekel, A ; Teyssier, R ; Cacciato, M ; Daddi, E ; Juneau, S ; Shankar, F
}

\begin{abstract}
Disk galaxies at high redshift have been predicted to maintain high gas surface densities due to continuous feeding by intense cold streams leading to violent gravitational instability, transient features, and giant clumps. Gravitational torques between the perturbations drive angular momentum out and mass in, and the inflow provides the energy for keeping strong turbulence. We use analytic estimates of the inflow for a self-regulated unstable disk at a Toomre stability parameter Q 1 , and isolated galaxy simulations capable of resolving the nuclear inflow down to the central parsec. We predict an average inflow rate $10 \mathrm{M}$ sun yr-1 through the disk of a $1011 \mathrm{M}$ sun galaxy, with conditions representative of z 2 stream-fed disks. The inflow rate scales with disk mass and $(1+\mathrm{z}) 3 / 2$. It includes clump migration and inflow of the smoother component, valid even if clumps disrupt. This inflow grows the bulge, while only a fraction of $>10-3$ of it needs to accrete onto a central black hole $(\mathrm{BH})$, in order to obey the observed $\mathrm{BH}$-bulge relation. A galaxy of $1011 \mathrm{M}$ sun at $\mathrm{z} 2$ is expected to host a $\mathrm{BH}$ of $108 \mathrm{M}$ sun, accreting on average with moderate sub-Eddington luminosity L X 1042-1043 erg s-1, accompanied by brighter episodes when dense clumps coalesce. We note that in rare massive galaxies at $\mathrm{z} 6$, the same process may feed $109 \mathrm{M}$ sun $\mathrm{BH}$ at the Eddington rate. High central gas column densities can severely obscure active galactic nuclei in high-redshift disks, possibly hindering their detection in deep X-ray surveys.
\end{abstract}

DOI: https://doi.org/10.1088/2041-8205/741/2/L33

Other titles: Black Hole growth and AGN obscuration by instability-driven inflows in high-redshift disk galaxies fed by cold streams

Posted at the Zurich Open Repository and Archive, University of Zurich

ZORA URL: https://doi.org/10.5167/uzh-54772

Journal Article

Published Version

Originally published at:

Bournaud, F; Dekel, A; Teyssier, R; Cacciato, M; Daddi, E; Juneau, S; Shankar, F (2011). Black hole growth and active galactic nuclei obscuration by instability-driven inflows in high-redshift disk galaxies fed by cold streams. Astrophysical Journal Letters, 741(2):L33.

DOI: https://doi.org/10.1088/2041-8205/741/2/L33 


\title{
BLACK HOLE GROWTH AND ACTIVE GALACTIC NUCLEI OBSCURATION BY INSTABILITY-DRIVEN INFLOWS IN HIGH-REDSHIFT DISK GALAXIES FED BY COLD STREAMS
}

\author{
Frédéric Bournaud ${ }^{1}$, Avishai Dekel $^{2}$, Romain Teyssier ${ }^{1,3}$, Marcello Cacciato $^{2}$, \\ Emanuele Daddi ${ }^{1}$, STÉPhanie Juneau $^{4}$, and Francesco Shankar ${ }^{5}$ \\ ${ }^{1}$ CEA, IRFU, SAp, 91191 Gif-sur-Yvette, France; frederic.bournaud@cea.fr \\ ${ }^{2}$ Racah Institute of Physics, The Hebrew University, Jerusalem 91904, Israel; dekel@phys.huji.ac.il \\ ${ }^{3}$ Institute for Theoretical Physics, University of Zürich, CH-8057 Zürich, Switzerland \\ ${ }^{4}$ Steward Observatory, University of Arizona, Tucson, AZ 85721, USA; sjuneau@ as.arizona.edu \\ ${ }^{5}$ Max-Planck-Institüt für Astrophysik, D-85748, Garching, Germany \\ Received 2011 July 7; accepted 2011 September 20; published 2011 October 20
}

\begin{abstract}
Disk galaxies at high redshift have been predicted to maintain high gas surface densities due to continuous feeding by intense cold streams leading to violent gravitational instability, transient features, and giant clumps. Gravitational torques between the perturbations drive angular momentum out and mass in, and the inflow provides the energy for keeping strong turbulence. We use analytic estimates of the inflow for a self-regulated unstable disk at a Toomre stability parameter $Q \sim 1$, and isolated galaxy simulations capable of resolving the nuclear inflow down to the central parsec. We predict an average inflow rate $\sim 10 M_{\odot} \mathrm{yr}^{-1}$ through the disk of a $10^{11} M_{\odot}$ galaxy, with conditions representative of $z \sim 2$ stream-fed disks. The inflow rate scales with disk mass and $(1+z)^{3 / 2}$. It includes clump migration and inflow of the smoother component, valid even if clumps disrupt. This inflow grows the bulge, while only a fraction of $\gtrsim 10^{-3}$ of it needs to accrete onto a central black hole $(\mathrm{BH})$, in order to obey the observed BH-bulge relation. A galaxy of $10^{11} M_{\odot}$ at $z \sim 2$ is expected to host a $\mathrm{BH}$ of $\sim 10^{8} M_{\odot}$, accreting on average with moderate sub-Eddington luminosity $L_{\mathrm{X}} \sim 10^{42}-10^{43} \mathrm{erg} \mathrm{s}^{-1}$, accompanied by brighter episodes when dense clumps coalesce. We note that in rare massive galaxies at $z \sim 6$, the same process may feed $\sim 10^{9} M_{\odot} \mathrm{BH}$ at the Eddington rate. High central gas column densities can severely obscure active galactic nuclei in high-redshift disks, possibly hindering their detection in deep X-ray surveys.
\end{abstract}

Key words: galaxies: active - galaxies: formation - galaxies: high-redshift - galaxies: nuclei - X-rays: galaxies

Online-only material: color figures

\section{INTRODUCTION}

The growth of massive black holes (BHs) and their associated active galactic nuclei (AGNs) are commonly assumed to be driven by gas inflows from galaxy mergers (Di Matteo et al. 2005; Hopkins et al. 2006). However, baryons are fed into highredshift galaxies through cold streams, in which the contribution of smooth gas and small clumps is larger than that of mergers of mass ratio greater than 1:10 (Dekel et al. 2009a; Brooks et al. 2009).

Cold accretion and high gas fractions in high-redshift disks trigger a violent instability, often characterized by giant starforming clumps. Using analytic estimates (Section 2) and simulations (Section 3), we address the possibility that the gas supply for $\mathrm{BH}$ growth is provided by instability-driven inflows and evaluate the obscuration of AGNs in this model (Section 4).

\section{INFLOW IN HIGH-REDSHIFT UNSTABLE DISKS}

Many $z \gtrsim 1$ galaxies of stellar masses $\approx 10^{10}-10^{11} M_{\odot}$ are rotating disks with giant clumps of $\sim 10^{8}-10^{9} M_{\odot}$ each (Elmegreen et al. 2007; Genzel et al. 2011). In most cases, ongoing mergers are not favored by gas kinematics (Genzel et al. 2008) and photometric properties (Elmegreen et al. 2009). Instead, high gas fractions of 50\% (Daddi et al. 2010; Tacconi et al. 2010) and high gas velocity dispersions (Förster Schreiber et al. 2009) are consistent with gravitational instability and selfregulation at $Q \sim 1$.

The occurrence and persistence of gravitational disk instability at high redshift is a natural result of the high surface density of the cold disk component, which is maintained by the continuous gas supply through cold cosmic streams (Dekel et al. 2009a). The instability is predicted to be self-regulated in steady state for a few Gyr (Dekel et al. 2009b, DSC09), and cosmological simulations reveal clumpy disks that resemble the observed ones (Agertz et al. 2009; Ceverino et al. 2010). If giant clumps survive stellar feedback for a few $10^{8} \mathrm{yr}$, they migrate into a central bulge (Noguchi 1999; Bournaud et al. 2007), as supported by observations (Genzel et al. 2008). Typical clumps seem to survive (Krumholz \& Dekel 2010; Genzel et al. 2011), while the most luminous clumps could undergo faster disruption (Murray et al. 2010; Genzel et al. 2011). Nevertheless, the inflow is a robust feature of the disk instability, driven by gravity torques independently of the survivability of bound clumps (Gammie 2001; DSC09).

A key element in the self-regulated instability at $Q \sim 1$, given the high surface density, is maintaining strong turbulence, with a velocity dispersion of $\sim 50 \mathrm{~km} \mathrm{~s}^{-1}$ for circular velocity $\sim 200 \mathrm{~km} \mathrm{~s}^{-1}$, as observed in high- $z$ disks. Supersonic turbulence dissipates in a few disk vertical crossing times (Mac Low 1999) and should therefore be continuously powered. An obvious source is the gravitational energy released when mass flows toward the center (DSC09; Elmegreen \& Burkert 2010). Assuming that the incoming streams deposit mass and energy at the outskirts of the disk where they do not contribute directly to driving disk turbulence, as is suggested by cosmological simulations (Agertz et al. 2009; Ceverino et al. 2010), the main energy source is the inflow within the disk (Krumholz \& Burkert 2010). This energy is transferred to velocity dispersion by gravitational torques involving massive clumps, transient features, 
Table 1

Simulation Parameters, Average Gas Inflow Rates $\dot{M}_{\mathrm{g}}$ Between $t=250$ and $400 \mathrm{Myr}$, and Predicted Value $f_{\mathrm{g}} \dot{M}_{\mathrm{b}}$ from Equation (2) in $M_{\odot} \mathrm{yr}^{-1}$

\begin{tabular}{lrccccccr}
\hline \hline ID & $\epsilon_{\text {AMR }}$ & $M_{*}\left(\times 10^{10} M_{\odot}\right)$ & $\begin{array}{c}R_{\mathrm{d}} \\
(\mathrm{kpc})\end{array}$ & $f_{\text {bulge }}$ & $\eta_{\mathrm{SN}}$ & $\dot{M}_{\mathrm{g}}(150 \mathrm{pc})$ & $\dot{M}_{\mathrm{g}}(1 \mathrm{kpc})$ & $f_{\mathrm{g}} \dot{M}_{\mathrm{b}}$ \\
\hline HR1 & $1.7 \mathrm{pc}$ & 7.0 & 16 & $14 \%$ & 0.5 & 5.6 & 7.2 & 15.0 \\
MR1 & $10.2 \mathrm{pc}$ & 7.0 & 16 & $14 \%$ & 0.5 & 5.2 & 6.8 & 15.0 \\
HR2 & $3.4 \mathrm{pc}$ & 1.5 & 7 & $8 \%$ & 0.5 & 1.2 & 2.9 & 3.2 \\
MR2 & $10.2 \mathrm{pc}$ & 1.5 & 7 & $8 \%$ & 0.5 & 1.3 & 3.1 & 3.2 \\
MR3 & $10.2 \mathrm{pc}$ & 3.3 & 12 & $0 \%$ & 0.5 & 3.4 & 6.5 & 8.2 \\
MR4 & $10.2 \mathrm{pc}$ & 7.0 & 16 & $14 \%$ & $0.5-5$ & 4.4 & 6.9 & 15.0 \\
\hline
\end{tabular}

and smoother regions of the disk. Stellar feedback is unlikely to be the main driver of the turbulence, e.g., because the velocity dispersion is not observed to be tightly correlated with proximity to star-forming clumps (Förster Schreiber et al. 2009; Genzel et al. 2011).

The baryonic inflow rate $\dot{M}_{\mathrm{b}}$ is estimated to be

$$
\dot{M}_{\mathrm{b}} \sim 0.2 \frac{M_{\mathrm{d}}}{t_{\mathrm{d}}}\left(\frac{\sigma}{V}\right)^{2},
$$

where $M_{\mathrm{d}}$ is the disk mass, $t_{\mathrm{d}}$ is the crossing time, $V$ is the circular velocity, and $\sigma$ is the one-dimensional velocity dispersion. At this rate, the power released by the inflow down the potential gradient, $\dot{M}_{\mathrm{b}} V^{2}$, compensates for the dissipative loss rate, $M_{\mathrm{g}} \sigma^{2} / t_{\mathrm{dis}}$, where $M_{\mathrm{g}} \sim 0.5 M_{\mathrm{d}}$ is the disk gas mass and $t_{\text {dis }} \sim 3 t_{\mathrm{d}}$ (M. Cacciato et al. 2011, in preparation). ${ }^{6}$ Similar estimates are obtained from the rate of energy exchange by clump encounters (DSC09, Equations (21) and (7)), and from the angular momentum exchange among the transient perturbations in a viscous disk (Gammie 2001; Genzel et al. 2008, DSC09, Equation (24)). Adopting $\sigma / V \sim 0.2$ and $t_{\mathrm{d}} \sim 30 \mathrm{Myr}$ at $z=2$, the inflow rate is

$$
\dot{M}_{\mathrm{b}} \simeq 25 M_{\mathrm{b}, 11}(1+z)_{3}^{3 / 2},
$$

where $M_{\mathrm{b}, 11}$ is the disk mass in $10^{11} M_{\odot}$ and $(1+z)_{3}=(1+z) / 3$. Note that the $(1+z)_{3}^{3 / 2}$ scaling corresponds to the disk crossing time, proportional to the halo crossing time at fixed overdensity in a matter-dominated universe.

A continuity equation for the disk gas, being drained into star formation, outflows, and bulge, and replenished by streams, yields at $z \leqslant 3$ a steady state with $M_{\mathrm{b}}$ about one-third of the cosmological baryonic accretion rate (Bouché et al. 2010; Krumholz \& Dekel 2011). With the latter being $\sim 80 M_{12}(1+$ $z)_{3}^{5 / 2} M_{\odot} \mathrm{yr}^{-1}$ (Neistein et al. 2006, $M_{12}$ being the halo mass in $10^{12} M_{\odot}$ ), this estimate is consistent with Equation (2) at $z=3$, with a different redshift dependence for the cosmological inflow rate.

\section{SIMULATIONS}

We perform six idealized simulations using the Adaptive Mesh Refinement (AMR) code RAMSES (Teyssier 2002), with a density-dependent grid refinement strategy and a barotropic cooling model producing realistic phase-space interstellar medium (ISM) structure (Bournaud et al. 2010). The initial parameters of the six simulations are listed in Table 1. The smallest cell size is $\epsilon_{\mathrm{AMR}}=1.7$ or $3.4 \mathrm{pc}$ in high-resolution (HR) runs and $10.2 \mathrm{pc}$ in medium-resolution (MR) runs. Cells are refined if they contain a gas mass larger than $2 \times 10^{3} M_{\odot}$, or more than 20 particles, or if the Jeans length is resolved by less than four

\footnotetext{
6 We used $0.5 / 3 \approx 0.2$ in Equation $(1)$.
}

cells. The initial numbers of dark matter and stellar particles are $4 \times 10^{6}$ and $6 \times 10^{5}$ in HR and MR, respectively. Star formation occurs above a density threshold of $5 \times 10^{4}$ and $4 \times 10^{3} \mathrm{~cm}^{-3}$ for $\mathrm{HR}$ and MR, with a star-formation efficiency per local free-fall time of $4 \%$. A fraction of the supernovae energy $\eta_{\mathrm{SN}}=0.5$ is released as kinetic feedback. For MR4, feedback is boosted up after $250 \mathrm{Myr}$ to $\eta_{\mathrm{SN}}=5$ in order to disrupt the clumps.

The simulations start as pre-formed disks in dark-matter halos. The exponential disk is truncated at $R_{\mathrm{d}}$, with scale length $R_{\mathrm{d}} / 4$ and $R_{\mathrm{d}} / 1.5$ for stars and gas, respectively. The darkmatter halo has a Burkert profile with a scale length $R_{\mathrm{d}} / 3$. The dark-matter mass fraction within $R_{\mathrm{d}}$ is 0.25 . The key parameter distinguishing high-redshift disks is the gas fraction, set to $50 \%$, as observed at $z \sim 2$. A small bulge containing a fraction $f_{\text {bulge }}$ of the stellar mass is assumed.

Within one orbital time, the violent gravitational instability, with $Q<1$ in the initial conditions, develops giant baryonic clumps and supersonic turbulence. The simulations resolve dense gas structures up to $\gtrsim 10^{7} \mathrm{~cm}^{-3}$, with a turbulence cascade in the ISM probed by the spectral analysis of model HR1 (Bournaud et al.2010, Section 3.4 and Figure 15). These isolated models allow us to follow the evolution of unstable disks for a couple of orbital times, i.e., a few $10^{8} \mathrm{yr}$, after which external gas supply should be included.

These simulations demonstrate that the instability-driven inflow is a generic feature, not limited to the migration of clumps. Figure 1 shows two snapshots of run HR1. At $t=$ $210 \mathrm{Myr}$, giant clumps have formed in the outer disk and the turbulent speed has reached a steady state with a mass-weighted average $\sigma \simeq 65 \mathrm{~km} \mathrm{~s}^{-1}$. About half of the gas mass is located between the clumps, the central kpc containing initially only $3 \times 10^{7} M_{\odot}$ of gas. At $t=330 \mathrm{Myr}$, the giant clumps have barely started their inward migration but the central kiloparsec already contains $1.5 \times 10^{9} M_{\odot}$ of originally diffuse gas that was driven in by torques.

Figure 2 shows the relative change in angular momentum per rotation period due to the gravity torques, $\Delta L / L=2 \pi F_{t} / F_{r}$, where $F_{t}$ and $F_{r}$ are the tangential and radial components of the force, averaged in radial bins. In the inner $4 \mathrm{kpc}$, most angular momentum is removed within one rotation, demonstrating the efficiency torquing driving the inflow.

Table 1 lists the gas inflow rates through spherical shells at $r=1 \mathrm{kpc}$ and $150 \mathrm{pc}$, averaged between $t=250$ and $450 \mathrm{Myr}$, i.e., between the onset of clump formation and their central coalescence. The simulated gas inflow rate at $1 \mathrm{kpc}$ is in the ballpark of the theoretical estimate of Equation (2), smaller by up to a factor of two, probably because the replenishment by fresh gas is ignored in the simulations. The inflow rate is not affected by the stronger feedback and clump disruption in run MR4. Indeed, in the simulation by Genel et al. (2011) in which the clumps are disrupted by enhanced outflows, the inflow rate is similar to that in their reference simulation where the clumps 


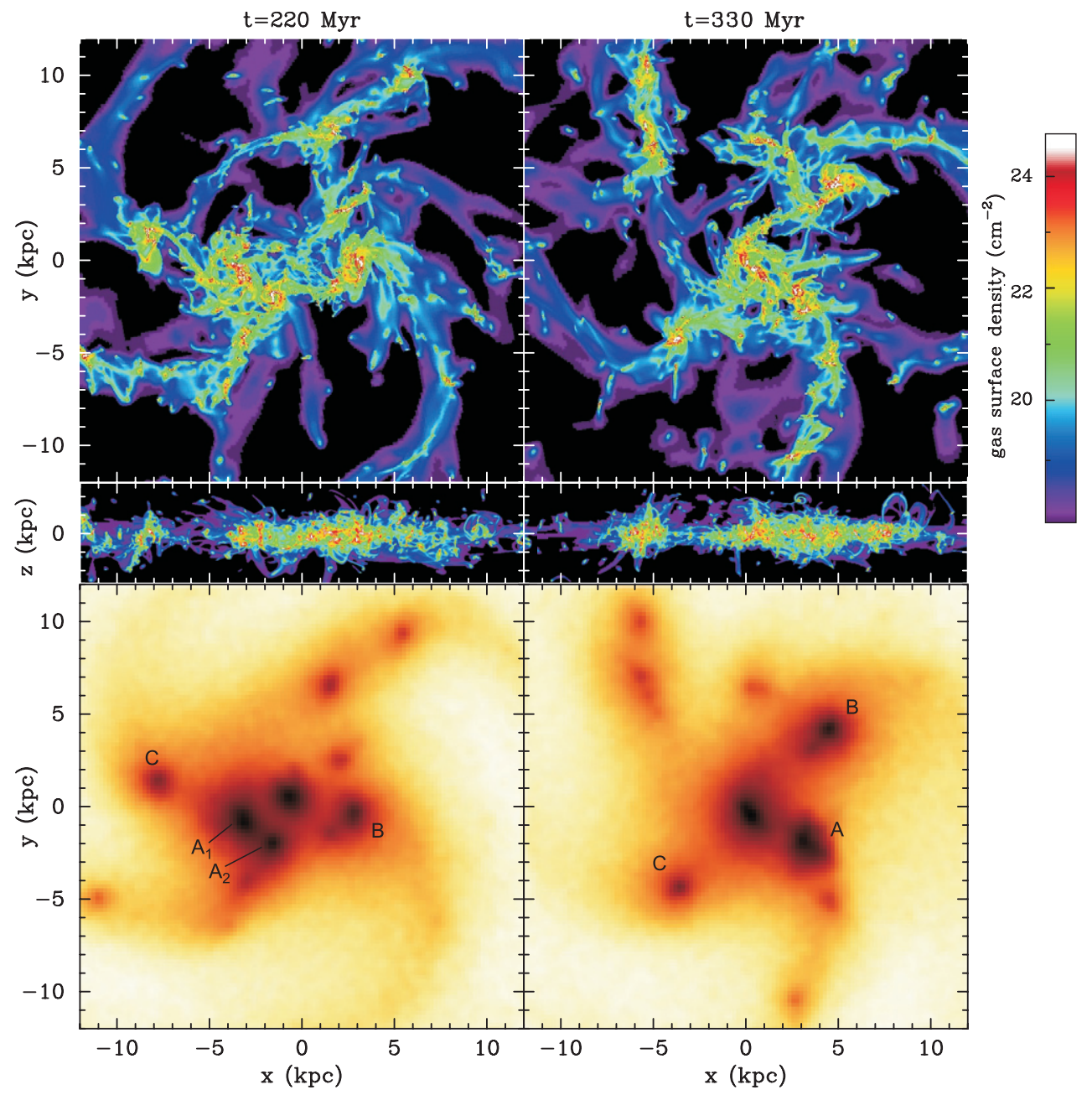

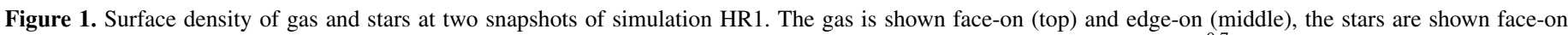

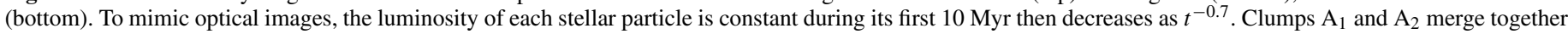
between the snapshots.

(A color version of this figure is available in the online journal.)

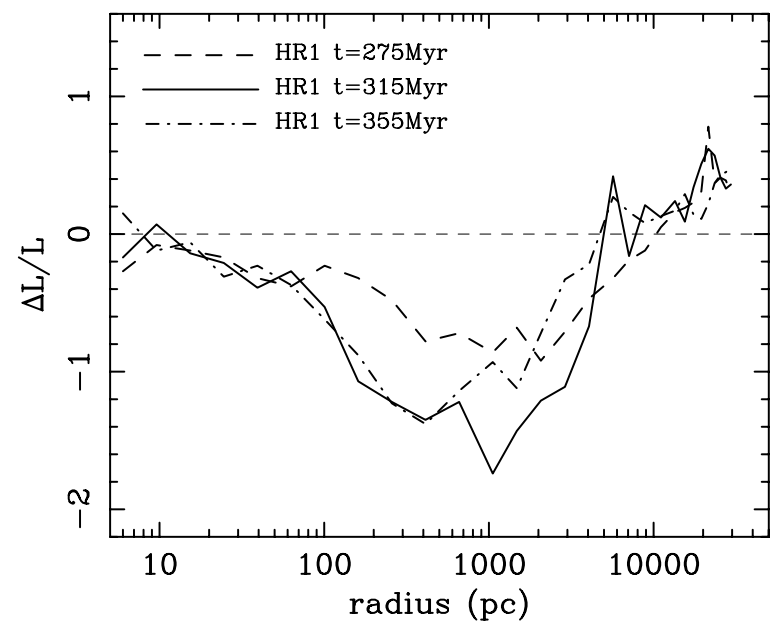

Figure 2. Relative change in angular momentum per rotation period at radius $r$ in the disk of run HR1, as evaluated from the gravitational torques.

survive, reaching in both cases a peak gas surface density higher than $10^{3} M_{\odot} \mathrm{pc}^{-2} \approx 10^{23} \mathrm{~cm}^{-2}$.

Figure 3 shows the net gas inflow rate across spherical shells about the stellar center of mass. Local inflow rates exceeding
$10 M_{\odot} \mathrm{yr}^{-1}$ are seen beyond the inner $1.5 \mathrm{kpc}$, dominated by clump migration. The inner kiloparsec is dominated by smooth inflow at a rate of $\sim 5 M_{\odot} \mathrm{yr}^{-1}$. The mild decline between $1 \mathrm{kpc}$ and $50 \mathrm{pc}$ can be partly explained by a geometry effect, as the sphere radius becomes smaller than the disk thickness. This is demonstrated by the inflow rate through cylindrical boundaries being rather constant with radius (Figure 3, bottom panel). A large fraction of the inflow takes place above and below the midplane in the thick gaseous disk. An inflow rate of $1-2 M_{\odot} \mathrm{yr}^{-1}$ nevertheless persists through the central few parsecs across spherical boundaries. We note that non-negligible outflows, presumably from stellar feedback, further reduce the net inflow rate in the central tens of parsecs. A comparison of runs HR1 and MR1 indicates no resolution effects on scales of 10-100 pc.

This gas inflow fuels star formation in the bulge. Galaxy HR1 at $t=300 \mathrm{Myr}$ has a total SFR of $132 M_{\odot} \mathrm{yr}^{-1}$, of which $16 M_{\odot} \mathrm{yr}^{-1}$ is in the central kpc. This is less than the SFR of 42 and $31 M_{\odot} \mathrm{yr}^{-1}$ in clumps $\mathrm{A}$ and $\mathrm{B}$, but comparable to the $14 M_{\odot} \mathrm{yr}^{-1}$ in clump C. At $t=450 \mathrm{Myr}$; the stellar bulge mass has grown from $9.8 \times 10^{9} M_{\odot}$ to $2.2 \times 10^{10} M_{\odot}$. The persistence of star formation in the bulge as a result of disk instability is consistent with observations showing that bulges in clumpy disks are bluer than bulges in smooth disks (Elmegreen et al. 2009). 


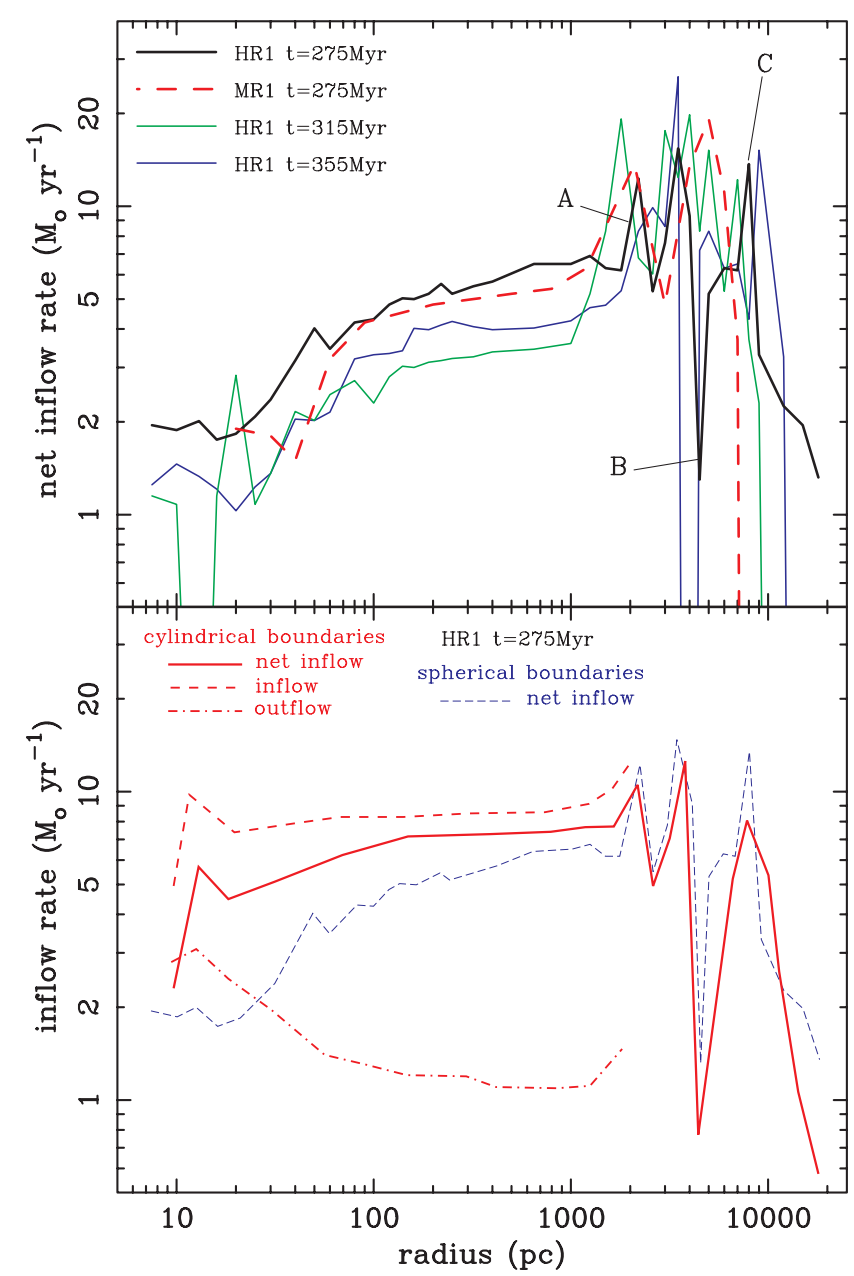

Figure 3. Radial profiles of the gas inflow rate. Top: net inflow (inflow minus outflow) through spherical boundaries for run HR1 at three different times and for run MR1. It is dominated by clump migration at large radii (note that clump B is moving outward) and by smooth inflow of 1-7 $M_{\odot} \mathrm{yr}^{-1}$ between $70 \mathrm{pc}$ and $2 \mathrm{kpc}$. Below $\sim 30-50 \mathrm{pc}$ the inflow rate drops, but it remains at $1-2 M_{\odot} \mathrm{yr}^{-1}$ in the inner $30 \mathrm{pc}$. Bottom: net inflow rate through cylindrical and spherical boundaries for HR1, comparing inflow and outflow. The drop in the net spherical inflow rate at small radii is dominated by the geometrical effect, and outflows further reduce the net inflow.

(A color version of this figure is available in the online journal.)

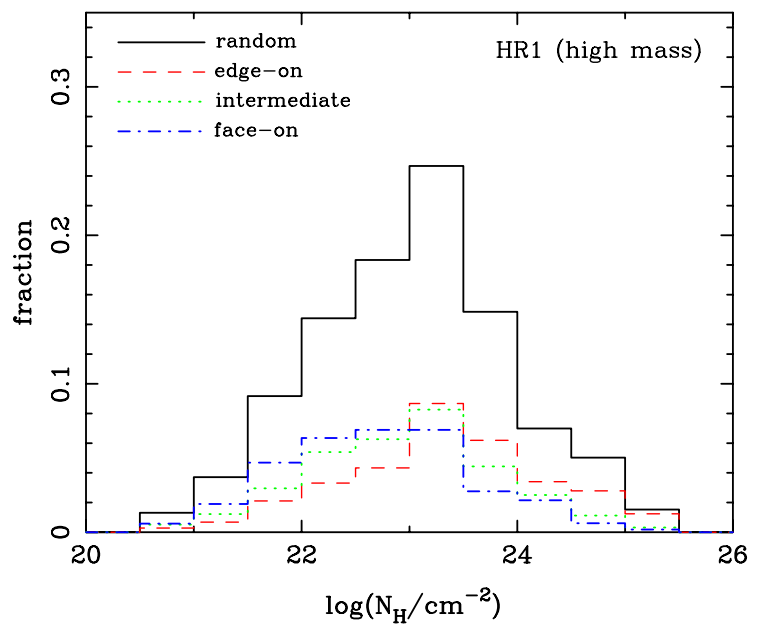

\section{AGN OBSCURATION IN GAS-RICH DISKS}

The central $\mathrm{BH}$ resides in a dense and thick gaseous disk (Figure 1), which could obscure an AGN. To quantify this, we computed the column densities over 60 random lines of sight through the centers of galaxy HR1 at $t=330 \mathrm{Myr}$ and HR2 at $t=380 \mathrm{Myr}$. The results are insensitive to the choice of center, selected to be either the center of mass of old stars or the point of maximum vorticity. The foreground hydrogen column density $N_{\mathrm{H}}$ was estimated over a cross-section corresponding to the minimum Jeans length, $4 \times \epsilon_{\mathrm{AMR}}$.

Figure 4 shows the distribution of $N_{\mathrm{H}}$ for runs HR1 and HR2 and for various sets of inclinations. Fifteen percent of the lines of sight reach Compton thickness, $\log \left(N_{\mathrm{H}}\right) \geqslant 24.1$, and $50 \%$ have $\log \left(N_{\mathrm{H}}\right) \geqslant 23$, i.e., strong obscuration. The obscuration tends to be higher in the more massive disk HR1 where Compton thickness can be reached even for face-on orientations owing to the $\sim 1 \mathrm{kpc}$ thickness of the gas disk. The lower-mass galaxy HR2, perhaps more representative at $z<2$, can reach Compton thickness almost exclusively for edge-on orientations.

If most gas in the central parsec lies in a torus, unresolved in our models, the obscuration on edge-on projections may be further enhanced and the dependence of obscuration on inclination may be stronger. Significant obscuration would still be present along most lines of sight, because the column densities measured in the simulations are on average not dominated by the central $10 \mathrm{pc}$.

\section{DISCUSSION: ACCRETION ONTO THE BLACK HOLE}

The isolated simulations presented here, using high gas fraction representative of $z \sim 2$ disks, have a disk inflow rate consistent with cosmological simulations of high-redshift stream-fed galaxies (Ceverino et al. 2010). They reach resolutions better than $2 \mathrm{pc}$, hence resolving the nuclear inflow down to scales at which other processes drive the actual small-scale $\mathrm{BH}$ accretion (Combes 2001). Only a fraction of the inflowing gas needs to make it all the way into the $\mathrm{BH}$. The issues are similar to the case of merger-driven fueling, namely, getting rid of angular momentum while avoiding excessive star formation and AGN feedback. This is beyond the scope of our paper, and we limit ourselves to heuristic estimates.

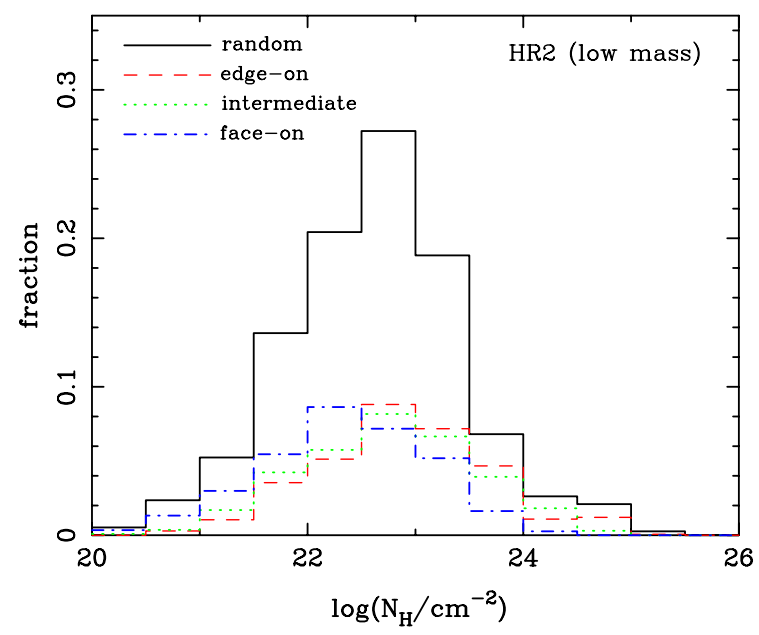

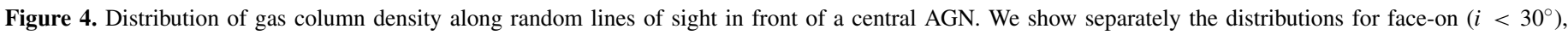
intermediate $\left(30^{\circ}<i<60^{\circ}\right)$, and edge-on $\left(i>60^{\circ}\right)$ orientations, the total fraction in each of these sub-samples being normalized to $33 \%$ for clarity.

(A color version of this figure is available in the online journal.) 
One can assume that the local relation between the $\mathrm{BH}$ mass $M_{\mathrm{BH}}$ and the bulge properties (mass $M_{\mathrm{blg}}$ and velocity dispersion $\sigma$ ) is crudely valid at high redshift. If we adopt $M_{\mathrm{BH}} / M_{\mathrm{blg}} \sim 10^{-3}$ at $z=0$, assume it scales as $\sigma^{2}$, and allow a cosmological scaling $\sigma^{2} \propto(1+z)$, then we obtain $M_{\mathrm{BH}} / M_{\mathrm{blg}} \sim 3 \times 10^{-3}$ at $z \sim 2$. A $z \sim 2$ galaxy of baryonic mass $10^{11} M_{\odot}$ hosts a $\mathrm{BH}$ of $\sim 10^{8} M_{\odot}$, while in a gravitationally unstable steady state it is typically half-bulge and half-disk (DSC09). According to Equation (2), the average inflow rate through the disk into the bulge is $M_{\mathrm{blg}} \sim 12.5 M_{\odot} \mathrm{yr}^{-1}$. If the ratio of average accretion rates into bulge and $\mathrm{BH}$ follow the ratio of the corresponding masses, the $\mathrm{BH}$ accretes on average at $\dot{M}_{\mathrm{BH}} \sim 0.04 M_{\odot} \mathrm{yr}^{-1}$, which is $1 \%-2 \%$ of the Eddington rate (assuming a 0.1 efficiency for mass-to-energy conversion). The corresponding bolometric luminosity is $2 \times 10^{44} \mathrm{erg} \mathrm{s}^{-1}$. With typically $1 \%-5 \%$ in X-rays, we estimate on average $L_{\mathrm{X}} \sim 10^{42}-10^{43} \mathrm{erg} \mathrm{s}^{-1}$, scaling with galaxy mass and with $(1+z)^{2.5}$. While the average luminosity would be modest, short episodes of higher accretion rate, possibly up to the Eddington level, occur during the central coalescence of migrating giant clumps-which could also bring with them seed BHs (Elmegreen et al. 2008a).

At very high redshift, the same process could feed brighter AGNs in rare massive systems (Di Matteo et al. 2011), provided that unstable disks indeed form there. The average inflow rate increases with redshift (Equation (2)), such that a $z=6-10$ disk can support continuous accretion at the Eddington rate in self-regulated quasar mode if $M_{\mathrm{BH}} / M_{\mathrm{d}}$ is $\sim 0.04-0.08$, for the same $\sim 10^{-3}$ fraction of the inflow rate accreting onto the $\mathrm{BH}$, and assuming outflows would not affect the cold gas streaming inward. Then a seed $\mathrm{BH}$ of $10^{5} M_{\odot}$ at $z \sim 10$ can grow exponentially by $10 e$-folds to $2 \times 10^{9} M_{\odot}$ at $z \sim 6$, possibly explaining very massive $\mathrm{BHs}$ growing in gas-rich systems at $z>5$ (Mortlock et al. 2011; Treister et al. 2011). The typical baryonic mass of the host galaxy is $\sim 10^{11} M_{\odot}$, and if the halo mass is a few times $10^{12} M_{\odot}$, the comoving number density of such bright $z>5$ AGNs would be $\sim 10^{-8}$ to $10^{-6} \mathrm{Mpc}^{-3}$.

\section{CONCLUSION}

Violent gravitational instability in high-redshift disk galaxies naturally leads to the growth of a bulge and a central $\mathrm{BH}$; it can both feed an AGN and obscure it. This stems from the developing picture where many star-forming galaxies at high redshift are rotating disks with high gas fractions continuously fed by cold streams. Such disks develop instability that involves transient perturbations and giant clumps. Gravitational torquing among these perturbations lead to mass inflow, which provides energy for maintaining the strong turbulence required for selfregulated instability at $Q \sim 1$. The cosmological inflow rate along cold streams sets an upper limit, but the actual inflow rate toward the nucleus is determined by the disk instability on galactic scales.

High-redshift disks are very different from nearby spirals, the key difference being the much higher gas fraction. The instability then operates on short dynamical timescales, and drives an intense inflow through the disk at the level of $\sim 10 M_{\odot} \mathrm{yr}^{-1} M_{\mathrm{b}, 11}(1+z)_{3}^{3 / 2}$. In low-redshift disks, the instability is a secular process limited to non-axisymmetric modes with considerably weaker torquing: bars are invoked in some AGN models (Fanidakis et al. 2011; Begelman et al. 2006) but need about 10 rotation periods to convey some gas inward (Bournaud et al. 2005). Gravitational torquing in high- $z$ disks is much more efficient and involves richer gas reservoirs.

Disk instability at $z \sim 2$ can thus funnel half of the disk gas toward the center in 2 Gyr. This is similar to the mass inflow in a major merger (Hopkins et al. 2006), but spread over a 10 times longer period, resulting in a lower average AGN luminosity, with higher duty cycle, and high obscuration. This process could dominate, as the gas mass in the relatively smooth cosmological streams is larger than that associated with mergers-less than $10 \%$ of the cosmological infall is in major mergers (Dekel et al. 2009a; L'Huillier et al. 2011). The main prediction is thus that many high- $z$ AGNs should be hosted by star-forming disk galaxies, composed of clumpy disks and growing spheroids. Many would be moderately luminous, detectable by Chandra deep surveys at $z=2$ if un-obscured, but the high expected obscuration makes their detectability dubious, especially for galaxies below $10^{11} M_{\odot}$. These $\mathrm{BHs}$ can grow inside classical bulges (Elmegreen et al. 2008b).

Observationally, the fraction of X-ray-detectable AGNs indeed declines with decreasing stellar mass (Xue et al. 2010). Many AGNs are highly absorbed, individually undetectable in X-rays (e.g., Daddi et al. 2007), and many of these obscured AGNs have moderate intrinsic luminosities and lie in star-forming galaxies (Juneau et al. 2011). Host galaxies of moderate-luminosity AGNs do not show an excess of majormerger signatures (Grogin et al. 2005; Gabor et al. 2009) and often have disky morphologies (Schawinski et al. 2011). Small mergers may also help feed the $\mathrm{BH}$ while leaving the disk intact (Shankar 2010), but they are a component of the cosmological streams and can be considered to be part of the inflow within the disk.

The violent instability phase should end when the cosmological accretion rate declines and the system becomes stellar dominated. We expect less massive galaxies to remain unstable for longer times, because they retain higher gas fractions-one of the aspects of the downsizing of star formation (e.g., Juneau et al. 2005). This can result from the regulation of gas consumption in smaller galaxies (Dekel \& Silk 1986; Krumholz $\&$ Dekel 2011) and from the continuation of cold accretion to lower redshift for lower-mass halos (Dekel \& Birnboim 2006). It induces an inverse gradient of gas fraction with galaxy mass (as observed, Kannappan 2004) and a downsizing in gravitational instability which could result in downsizing of BH growth. This is a longer growth phase into later redshifts in lower-mass galaxies, with $\sim 10^{11} M_{\odot}$ galaxies growing BHs mostly at $z>1$ in their unstable disk phase, while clumpy disks of $\sim 10^{10} M_{\odot}$ may show AGN activity even after $z \sim 1$.

Simulations were performed at CCRT and TGCC under GENCI allocation 2011-GEN2192. We acknowledge discussions with Françoise Combes, Bruce Elmegreen, Tiziana Di Matteo, James Mullaney, a constructive referee report, and support from grants ERC-StG-257720 (F.B., S.J.), CosmoComp ITN (F.B., R.T., S.J.), ISF 6/08, GIF G-1052-104.7/2009, NSF AST-1010033, and a DIP grant (A.D.).

\section{REFERENCES}

Agertz, O., Teyssier, R., \& Moore, B. 2009, MNRAS, 397, L64 Begelman, M. C., Volonteri, M., \& Rees, M. J. 2006, MNRAS, 370, 289 Bouché, N., Dekel, A., Genzel, R., et al. 2010, ApJ, 718, 1001

Bournaud, F., Combes, F., \& Semelin, B. 2005, MNRAS, 364, L18 Bournaud, F., Elmegreen, B. G., \& Elmegreen, D. M. 2007, ApJ, 670, 237 
Bournaud, F., Elmegreen, B. G., Teyssier, R., Block, D. L., \& Puerari, I 2010, MNRAS, 409, 1088

Brooks, A. M., Governato, F., Quinn, T., Brook, C. B., \& Wadsley, J. 2009, ApJ, 694, 396

Ceverino, D., Dekel, A., \& Bournaud, F. 2010, MNRAS, 404, 2151

Combes, F. 2001, in Advanced Lectures on the Starburst-AGN Connection, Proceedings of a Conference held in Tonantzintla, Puebla, Mexico, 2000 June 26-30, ed. I. Aretxaga, D. Kunth, \& R. Mújica (Singapore: World Scientific), 223

Daddi, E., Alexander, D. M., Dickinson, M., et al. 2007, ApJ, 670, 173

Daddi, E., Bournaud, F., Walter, F., et al. 2010, ApJ, 713, 686

Dekel, A., \& Birnboim, Y. 2006, MNRAS, 368, 2

Dekel, A., Birnboim, Y., Engel, G., et al. 2009a, Nature, 457, 451

Dekel, A., Sari, R., \& Ceverino, D. 2009b, ApJ, 703, 785 (DSC09)

Dekel, A., \& Silk, J. 1986, ApJ, 303, 39

Di Matteo, T., Khandai, N., DeGraf, C., et al. 2011, arXiv:1107.1253

Di Matteo, T., Springel, V., \& Hernquist, L. 2005, Nature, 433, 604

Elmegreen, B. G., Bournaud, F., \& Elmegreen, D. M. 2008a, ApJ, 684, 829

Elmegreen, B. G., Bournaud, F., \& Elmegreen, D. M. 2008b, ApJ, 688, 67

Elmegreen, B. G., \& Burkert, A. 2010, ApJ, 712, 294

Elmegreen, B. G., Elmegreen, D. M., Fernandez, M. X., \& Lemonias, J. J. 2009, ApJ, 692, 12

Elmegreen, D. M., Elmegreen, B. G., Ravindranath, S., \& Coe, D. A. 2007, ApJ, 658,763

Fanidakis, N., Baugh, C. M., Benson, A. J., et al. 2011, MNRAS, 410, 53

Förster Schreiber, N. M., Genzel, R., Bouché, N., et al. 2009, ApJ, 706, 1364

Gabor, J. M., Impey, C. D., Jahnke, K., et al. 2009, ApJ, 691, 705

Gammie, C. F. 2001, ApJ, 553, 174
Genel, S., Naab, T., Genzel, R., et al. 2011, ApJ, submitted (arXiv:1011.0433) Genzel, R., Burkert, A., Bouché, N., et al. 2008, ApJ, 687, 59

Genzel, R., Newman, S., Jones, T., et al. 2011, ApJ, 733, 101

Grogin, N. A., Conselice, C. J., Chatzichristou, E., et al. 2005, ApJ, 627, L97

Hopkins, P. F., Hernquist, L., Cox, T. J., et al. 2006, ApJS, 163, 1

Juneau, S., Dickinson, M., Alexander, D. M., \& Salim, S. 2011, ApJ, 736, 104

Juneau, S., Glazebrook, K., Crampton, D., et al. 2005, ApJ, 619, L135

Kannappan, S. J. 2004, ApJ, 611, L89

Krumholz, M., \& Burkert, A. 2010, ApJ, 724, 895

Krumholz, M. R., \& Dekel, A. 2010, MNRAS, 406, 112

Krumholz, M. R., \& Dekel, A. 2011, ApJ, submitted (arXiv:1106.0301)

L'Huillier, B., Combes, F., \& Semelin, S. 2011, A\&A, submitted (arXiv:1108.4247)

Mac Low, M.-M. 1999, ApJ, 524, 169

Mortlock, D. J., Warren, S. J., Venemans, B. P., et al. 2011, Nature, 474, 616

Murray, N., Quataert, E., \& Thompson, T. A. 2010, ApJ, 709, 191

Neistein, E., van den Bosch, F. C., \& Dekel, A. 2006, MNRAS, 372, 933

Noguchi, M. 1999, ApJ, 514, 77

Schawinski, K., Treister, E., Urry, C. M., et al. 2011, ApJ, 727, L31

Shankar, F. 2010, in IAU Symp. 267, Co-Evolution of Central Black Holes and Galaxies, ed. B. Peterson, R. Somerville, \& T. Storchi-Bergmann (Cambridge: Cambridge Univ. Press), 248

Tacconi, L. J., Genzel, R., Neri, R., et al. 2010, Nature, 463, 781

Teyssier, R. 2002, A\&A, 385, 337

Treister, E., Schawinski, K., Volonteri, M., Natarajan, P., \& Gawiser, E. 2011, Nature, 474, 356

Xue, Y. Q., Brandt, W. N., Luo, B., et al. 2010, ApJ, 720, 368 